\title{
Evolution of supersonic downflows in a sunspot
}

\author{
C. J. Nelson ${ }^{1}$, S. Krishna Prasad ${ }^{1,2}$, and M. Mathioudakis ${ }^{1}$ \\ 1 Astrophysics Research Centre (ARC), School of Mathematics and Physics, Queen's University, Belfast BT7 1NN, UK \\ e-mail: c.nelson@qub.ac.uk \\ ${ }^{2}$ Centre for mathematical Plasma Astrophysics (CmPA), KU Leuven, Celestijnenlaan 200B, 3001 Leuven, Belgium
}

Received 19 December 2019 / Accepted 4 March 2020

\begin{abstract}
Context. Supersonic downflows have been observed in transition region spectra above numerous sunspots, however, little research has been conducted to date into how persistent these signatures are within sunspots on timescales longer than a few hours.

Aims. We aim to analyse the lead sunspot of AR 12526 to infer the properties and evolution of supersonic downflows occurring within it using high-spatial and spectral resolution data.

Methods. We analysed 16 large, dense raster scans sampled by the Interface Region Imaging Spectrograph. These rasters tracked the lead sunspot of AR 12526 across the solar disc at discrete times between 27 March 2016 and 2 April 2016, providing spectral profiles from the Si IV, O IV, Mg II, and C II lines. Additionally, we studied one sit-and-stare observation acquired on 1 April 2016 centred on the sunspot to analyse the evolution of supersonic downflows on shorter timescales.

Results. Supersonic downflows are variable within this sunspot both in terms of spatial structuring and velocities. Thirteen of the 16 raster scans display some evidence of supersonic downflows in the Si IV $1394 \AA$ line co-spatial to a sustained bright structure detected in the $1400 \AA$ slit-jaw imaging channel; a peak velocity of $112 \mathrm{~km} \mathrm{~s}^{-1}$ is recorded on 29 March 2016 . Evidence for supersonic downflows in the OIV $1401 \AA$ line is found in 14 of these rasters; the spatial structuring in this line often differs from that inferred from the Si IV $1394 \AA$ line. Only one example of a supersonic downflow is detected in the C II $1335 \AA$ line and no downflows are found in the $\mathrm{Mg}$ II $2796 \AA$ lines at these locations. In the sit-and-stare observations, no dual flow is initially detected, however, a supersonic downflow develops after approximately $60 \mathrm{~min}$. This downflow accelerates from $73 \mathrm{~km} \mathrm{~s}^{-1}$ to close to $80 \mathrm{~km} \mathrm{~s}^{-1}$ in both the Si IV $1394 \AA$ and O IV $1401 \AA$ lines over the course of 20 min before the end of the observation.

Conclusions. Supersonic downflows are found in the Si IV $1394 \AA$ line in 13 of the 16 rasters studied in this work. The morphology of these downflows evolved over the course of both hours and days and was often different in the Si IV $1394 \AA$ and O IV $1401 \AA$ lines. These events were found co-spatial to a bright region in the core of the Si IV $1394 \AA$ line, which appeared to form at the footpoints of coronal fan loops. Our results indicate that one raster is not enough to conclusively draw inferences about the properties of supersonic downflows within a sunspot during its lifetime.
\end{abstract}

Key words. sunspots - Sun: atmosphere - Sun: transition region - Sun: oscillations

\section{Introduction}

The solar atmosphere above sunspots is highly dynamic, with a range of transient events and oscillations being reported in the literature (for reviews see e.g. Solanki 2003; Khomenko $\&$ Collados 2015). These strong regions of vertical magnetic field (Hale 1908) act as ideal conduits along which magnetohydrodynamic (MHD) waves can propagate from the lower solar atmosphere into the upper solar atmosphere (examples include Fludra 2001; Rouppe van der Voort et al. 2003; Jess et al. 2012; Sych \& Nakariakov 2014) potentially depositing their energy to heat the local corona. It is important to note, however, that not everything within umbrae is always moving upwards. It is now known that downflowing plasma can play an important role in sunspot dynamics, having been associated with strong shocks in both the chromosphere (Henriques et al. 2017; Nelson et al. 2017) and transition region (as discussed by e.g. Dere 1982; Brynildsen et al. 2001, 2004; Kleint et al. 2014).

Dynamic shocks in the solar chromosphere, such as umbral flashes, have been linked to upflowing plasma for decades (see, for example, Beckers \& Tallant 1969; Wittmann 1969; Rouppe van der Voort et al. 2003). However, recent non-local thermodynamical equilibrium (LTE) modelling of chromospheric lines, using inversion codes such as the Non-LTE Inversion Code based on the Lorien Engine (NICOLE; Socas-Navarro et al. 2015), has indicated that such events could be formed by a blend of an upwardly propagating wave on a strongly downflowing background (as discussed by Henriques et al. 2017; Bose et al. 2019). Interestingly, such a model could also be invoked to simultaneously explain other events in the chromospheres of sunspots including small-scale umbral brightenings (Nelson et al. 2017) and short dynamic fibrils (Rouppe van der Voort \& de la Cruz Rodríguez 2013). Downflows have also been observed above rapidly evolving pores using the He I $10830 \AA$ line (Lagg et al. 2007).

Supersonic downflows in the transition region above sunspots have been of interest to the community ever since their discovery (see, for example, Dere 1982; Nicolas et al. 1982; Kjeldseth-Moe et al. 1988; Brynildsen et al. 2001, 2004; Tian et al. 2014a; Kleint et al. 2014; Samanta et al. 2018). These "dual flows", which were first detected as strongly redshifted secondary emission peaks in transition region spectra, form in localised regions of both the umbra and penumbra (as shown by the recent statistical analysis of Samanta et al. 2018). It has been suggested that these supersonic downflows are associated with light bridges in the lower solar atmosphere, 
Table 1. Details of the 16 raster scans analysed in this work.

\begin{tabular}{|c|c|c|c|c|c|c|c|c|c|c|c|}
\hline Date & Start (UT) & End (UT) & $x c^{\prime \prime}$ & $y c^{\prime \prime}$ & $\operatorname{Pix}_{x}$ & $\operatorname{Pix}_{y}$ & Si IV $1394 \AA$ & O IV $1401 \AA$ & C II $1335 \AA$ & $n_{\mathrm{p}, \mathrm{Si}}$ & $n_{\mathrm{p}, \mathrm{O}}$ \\
\hline $2016-03-27$ & 17:59:11 & 19:00:25 & -595 & 18 & 313 & 324 & $98 \mathrm{~km} \mathrm{~s}^{-1}$ & $99 \mathrm{~km} \mathrm{~s}^{-1}$ & No & 62 & 127 \\
\hline 2016-03-29 & 01:29:12 & $02: 30: 25$ & -323 & 29 & 302 & 307 & $72 \mathrm{~km} \mathrm{~s}^{-1}$ & $72 \mathrm{~km} \mathrm{~s}^{-1}$ & No & 25 & 55 \\
\hline 2016-03-29 & $06: 13: 11$ & $07: 14: 25$ & -304 & 16 & 384 & 369 & $107 \mathrm{~km} \mathrm{~s}^{-1}$ & $108 \mathrm{~km} \mathrm{~s}^{-1}$ & No & 73 & 122 \\
\hline 2016-03-29 & 09:29:11 & $10: 30: 25$ & -268 & 16 & 375 & 361 & $112 \mathrm{~km} \mathrm{~s}^{-1}$ & N/A & No & 11 & 56 \\
\hline 2016-03-29 & 15:19:11 & $16: 20: 25$ & -215 & 26 & 319 & 354 & $94 \mathrm{~km} \mathrm{~s}^{-1}$ & N/A & No & 61 & 136 \\
\hline $2016-03-30$ & 01:09:11 & $02: 10: 25$ & -126 & 19 & 358 & 363 & $89 \mathrm{~km} \mathrm{~s}^{-1}$ & $87 \mathrm{~km} \mathrm{~s}^{-1}$ & No & 16 & 65 \\
\hline 2016-03-30 & 08:19:09 & 09:20:23 & -58 & 28 & 346 & 354 & $94 \mathrm{~km} \mathrm{~s}^{-1}$ & $86 \mathrm{~km} \mathrm{~s}^{-1}$ & No & 2 & 6 \\
\hline $2016-03-30$ & 19:04:39 & $20: 05: 53$ & 47 & 25 & 356 & 343 & $85 \mathrm{~km} \mathrm{~s}^{-1}$ & $88 \mathrm{~km} \mathrm{~s}^{-1}$ & $100 \mathrm{~km} \mathrm{~s}^{-1}$ & 75 & 60 \\
\hline 2016-03-31 & 01:29:09 & $02: 30: 23$ & 109 & 27 & 341 & 331 & $76 \mathrm{~km} \mathrm{~s}^{-1}$ & $85 \mathrm{~km} \mathrm{~s}^{-1}$ & No & 64 & 57 \\
\hline 2016-03-31 & 08:34:11 & $09: 35: 25$ & 171 & 25 & 340 & 338 & $87 \mathrm{~km} \mathrm{~s}^{-1}$ & $85 \mathrm{~km} \mathrm{~s}^{-1}$ & No & 24 & 6 \\
\hline 2016-03-31 & $14: 24: 41$ & $15: 25: 55$ & 222 & 29 & N/A & N/A & N/A & N/A & N/A & 0 & 0 \\
\hline 2016-03-31 & 19:22:11 & $20: 23: 25$ & 266 & 22 & N/A & N/A & N/A & N/A & N/A & 0 & 0 \\
\hline 2016-04-01 & $05: 50: 11$ & $06: 51: 25$ & 355 & 40 & 297 & 330 & $85 \mathrm{~km} \mathrm{~s}^{-1}$ & $69 \mathrm{~km} \mathrm{~s}^{-1}$ & No & 18 & 12 \\
\hline 2016-04-01 & $14: 52: 13$ & $15: 53: 27$ & 428 & 32 & 294 & 341 & $71 \mathrm{~km} \mathrm{~s}^{-1}$ & N/A & No & 9 & 14 \\
\hline 2016-04-01 & $21: 20: 11$ & $22: 21: 25$ & 485 & 9 & 353 & 327 & $57 \mathrm{~km} \mathrm{~s}^{-1}$ & $60 \mathrm{~km} \mathrm{~s}^{-1}$ & No & 7 & 20 \\
\hline 2016-04-02 & $02: 41: 11$ & $03: 42: 25$ & 532 & 1 & 384 & 304 & $\mathrm{~N} / \mathrm{A}$ & $61 \mathrm{~km} \mathrm{~s}^{-1}$ & N/A & 0 & 5 \\
\hline
\end{tabular}

Notes. Columns are: date of raster; start time of raster; end time of raster; $x$-coordinate of the centre of the raster; $y$-coordinate of the centre of the raster; $x$-pixel, where the largest supersonic downflow was inferred from the Si IV $1394 \AA$ line; $y$-pixel, where the largest supersonic downflow was inferred from the Si IV $1394 \AA$ line; peak downflow velocity calculated from a double Gaussian fitting of the Si IV $1394 \AA$ Aine; downflow velocity in the O IV $1401 \AA$ line co-spatial to the peak Si IV $1394 \AA$ velocity (except the bottom row where the peak O IV $1401 \AA$ velocity is reported); downflow velocity in the C II $1335 \AA$ channel co-spatial to the peak Si IV $1394 \AA$ velocity; number of pixels displaying downflows in the Si IV $1394 \AA$ line; and number of pixels displaying downflows in the O IV $1401 \AA$ line.

however, the details of such a relationship are currently unknown (Nicolas et al. 1982). The extensive availability of high-spectral, temporal, and spatial resolution data since the launch of the Interface Region Imaging Spectrograph (IRIS; De Pontieu et al. 2014) has allowed for improved understanding of these events in recent years (for a recent review see Tian et al. 2018).

It is now thought possible that at least two different types of dual flows may exist in the transition region. Bursty, shortlived (of the order seconds) downflows with velocities of up to $200 \mathrm{~km} \mathrm{~s}^{-1}$ have been detected and associated with impulsive coronal rain (see, for example, Kleint et al. 2014). Typically, these events appear to have signatures in a wide complement of IRIS diagnostics, including the Mg II, C II, and Si IV lines. Of more relevance, potentially, are downflows which appear to have lifetimes of the order of minutes to hours and typical velocities of around $100 \mathrm{~km} \mathrm{~s}^{-1}$ (as discussed by e.g. Straus et al. 2015; Chitta et al. 2016). These events, which are potentially linked to siphon flows, are generally observed in the Si IV and O IV lines with only occasional signatures in the $\mathrm{Mg}$ II and C II lines (Samanta et al. 2018). Although these events have sometimes been observed to be stable over timescales of the order of hours, it is likely that they evolve significantly over the course of several days (Kjeldseth-Moe et al. 1988). Interestingly, the inferred downflow velocity from the O IV lines has sometimes been found to be around $10 \mathrm{~km} \mathrm{~s}^{-1}$ lower than the velocity calculated from the Si IV lines potentially implying the presence of multi-thermal, multi-threaded loops (Dere 1982; Nicolas et al. 1982; Chitta et al. 2016). It should be noted, however, that such a disparity in the downflow speeds measured from these spectral lines is not always present (Samanta et al. 2018).

In this article, we analyse the evolution of supersonic downflows within the lead sunspot of AR 12526 over the course of one week using high-resolution IRIS data. Of specific interest is understanding how persistent such downflows are within the sunspot, over both short and long timescales. Our work is set out as follows: In Sect. 2 we introduce the satellite data studied; in Sect. 3 we present our results, including analysis of both raster data and sit-and-stare observations; in Sect. 4 we present a discussion and our conclusions; and finally, in Sect. 5 we provide a brief summary.

\section{Observations}

Initially, 16400 -step dense $\left(0.35^{\prime \prime}\right.$ step-size) rasters covering AR 12526 at discrete times between 27 March 2016 and 2 April 2016 were studied. For each of these datasets the slit length was $175^{\prime \prime}$, the exposure time was $8 \mathrm{~s}$ (with a step cadence of $9.2 \mathrm{~s}$ ), and the spectral samplings were $0.025 \AA$ and $0.05 \AA$ for the far-ultraviolet (FUV) and near-ultraviolet (NUV) channels, respectively. During the rasters, the Slit-Jaw Imager (SJI) sampled each of the $1330 \AA, 1400 \AA, 2796 \AA$, and $2832 \AA$ channels sequentially, providing 100 images per raster for each filter. Each SJI image sampled a $167^{\prime \prime} \times 175^{\prime \prime}$ field of view (FOV) which tracked the IRIS slit, giving a total FOV of $307^{\prime \prime} \times 175^{\prime \prime}$. The cadence of each of the slit-jaw channels was approximately $37 \mathrm{~s}$ and the pixel scale was $0.33^{\prime \prime}$. The basic information for each of these datasets is included for reference in the first five columns of Table 1. The OBS ID for each raster was 3600108078 .

To supplement the raster data, we also analysed one sitand-stare sequence sampled by IRIS between 16:23:28 UT and 17:44:22 UT on 1 April 2016. During this time, the slit passed north-south through the umbra of the lead sunspot within AR 12526 at coordinates of $x_{\mathrm{c}}=480^{\prime \prime}, y_{\mathrm{c}}=34^{\prime \prime}$ (centre of the slit). These data had a $15 \mathrm{~s}$ exposure time (giving a cadence of $16.7 \mathrm{~s}$ ) returning a total of 290 spectra. The slit length was $119^{\prime \prime}$ and the spectral sampling in both the NUV and FUV channels was approximately $0.025 \AA$. The SJI sampled a $119^{\prime \prime} \times 119^{\prime \prime}$ FOV with the $1400 \AA$ and $2796 \AA$ channels sequentially, meaning each filter had a cadence of approximately $33 \mathrm{~s}$. The pixel scale of 


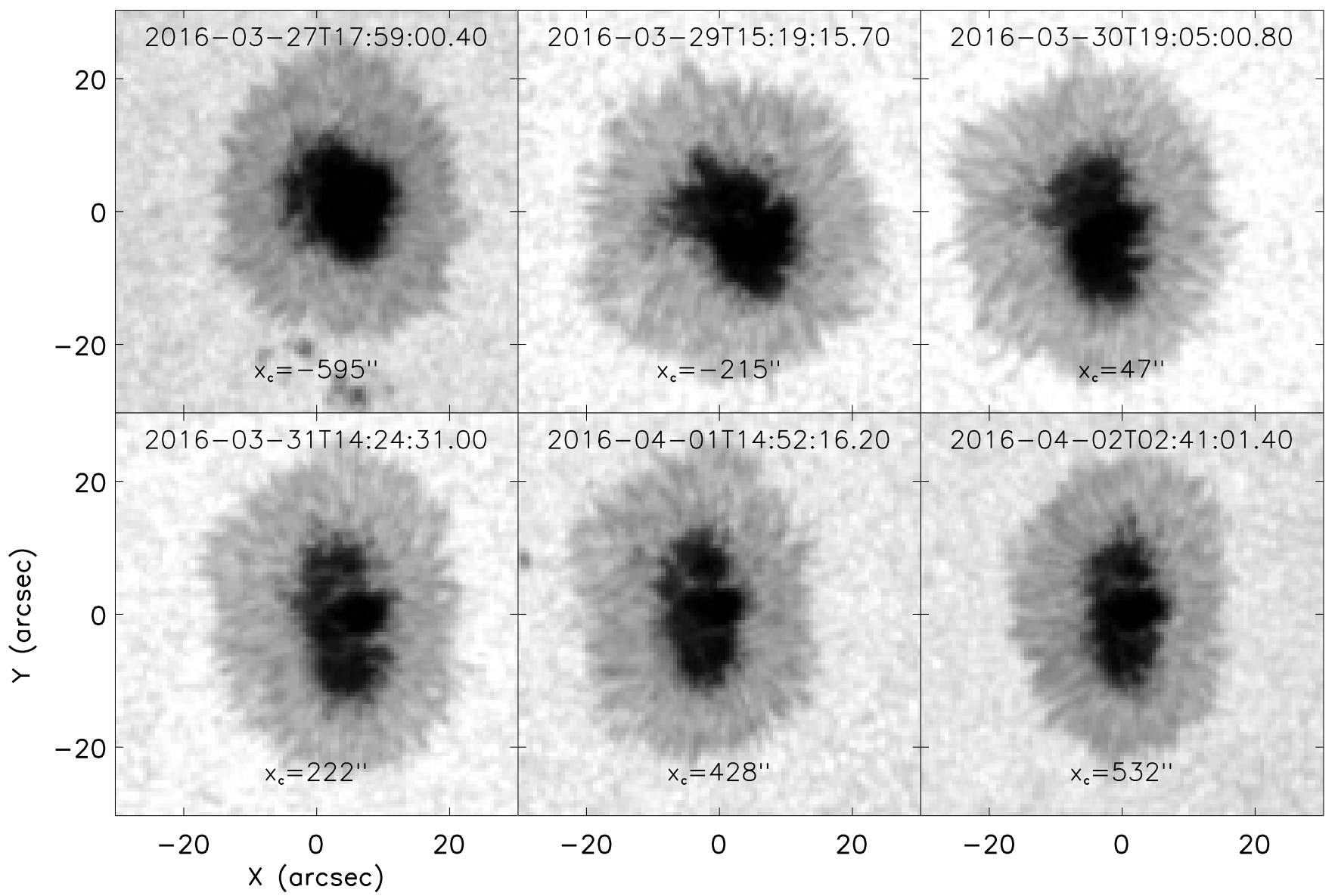

Fig. 1. Context imaging from the SDO/HMI continuum channel of the sunspot within AR 12526 over the course of the seven days analysed. Initially, no light bridge was evident, however, by 31 March 2016 one large light bridge had developed in the northern region of the sunspot. A second light bridge was also present in the southern portion of the sunspot, however, this was smaller and less obvious than the northern light bridge. The times and co-ordinates overlaid on each panel indicate the start time and centre position in the $x$-direction of the corresponding IRIS raster.

these SJI data was around $0.17^{\prime \prime}$. The OBS ID for this sit-andstare sequence was 3680261403 . The wavelength calibration for the FUV and NUV channels was confirmed for all IRIS datasets through analysis of the neutral O I $1355.6 \AA$ and Ni I $2799.5 \AA$ lines.

Finally, we studied data from the Solar Dynamics Observatory's Helioseismic and Magnetic Imager (SDO/HMI; Scherrer et al. 2012) and Atmospheric Imaging Assembly (SDO/AIA; Lemen et al. 2012) to give photospheric and coronal context to our analysis. Single line-of-sight (LOS) magnetic field maps and continuum intensity images from the SDO/HMI instrument co-temporal to the start of each raster and sit-and-stare sequence were downloaded and reduced; the post-reduction pixel scale was $0.6^{\prime \prime}$ and the cadence was $45 \mathrm{~s}$. Additionally, SDO/AIA $304 \AA$, $171 \AA$, and $193 \AA$ filter images were downloaded for the time-period corresponding to the entire sit-andstare sequence; the post-reduction pixel scales and cadences were $0.6^{\prime \prime}$ and $12 \mathrm{~s}$, respectively. A single SDO/AIA $1600 \AA$ image co-temporal to the start of the sit-and-stare sequence was also downloaded for alignment purposes. Initial alignment between all instruments was accomplished to the leading order using header information before more accurate alignments were completed by visually matching features in the SDO/HMI continuum, SDO/AIA $1600 \AA$, and IRIS SJI MgII $2796 \AA$ channels.

\section{Results}

\subsection{Raster scans}

We begin our analysis by providing a brief overview of the general properties of the sunspot studied in this work through time. In Fig. 1, we plot the sunspot as sampled by the SDO/HMI continuum filter at the start of six of the raster scans detailed in Table 1, one for each day in which a raster exits. On 27 March 2016, no light bridge is evident within the umbra of what appears to be a relatively circular sunspot. Over the course of the next three days, however, the sunspot becomes more elongated in the north-south direction before two distinct light bridges develop on 31 March 2016. Both light bridges have an east-west orientation with one forming in the north and one forming in the south, essentially splitting the umbra into three equal portions. The northern light bridge has a higher contrast than the southern light bridge, making it more evident in the SDO/HMI images plotted in this work. Both light bridges are present up to the time of the final raster at 02:41 UT on 2 April 2016.

In order to investigate whether supersonic downflows were present in the transition region of the sunspot during these scans, we performed double Gaussian fitting to the Si IV $1394 \AA$ and O IV $1401 \AA$ spectra (using gauss_fit.pro) on each pixel around the sunspot between velocities of $-45 \mathrm{~km} \mathrm{~s}^{-1}$ and $+175 \mathrm{~km} \mathrm{~s}^{-1}$. For the SiIV $1394 \AA$ line, we considered that a supersonic 


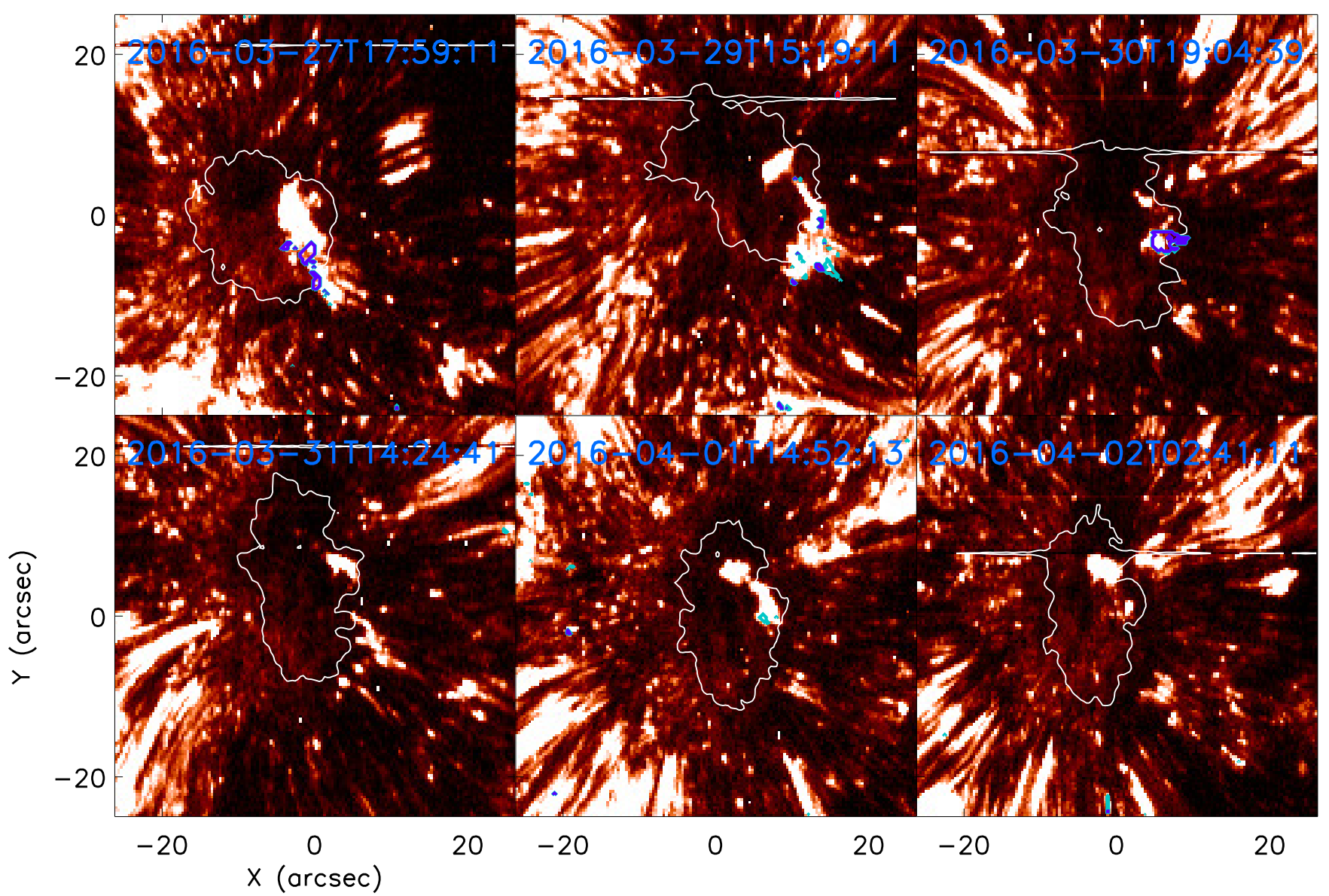

Fig. 2. Intensity at the rest wavelength of the Si IV $1394 \AA$ line in the sunspot from the rasters corresponding to the SDO/HMI continuum panels in Fig. 1. The overlaid coloured contours highlight regions where double Gaussian fitting indicated the presence of supersonic downflows (aqua and blue correspond to $50 \mathrm{~km} \mathrm{~s}^{-1}$ and $75 \mathrm{~km} \mathrm{~s}^{-1}$, respectively). All supersonic downflows within the sunspot are co-spatial to a sustained region of increased intensity in the line core. Again, pointing coordinates are not relative and are only provided for an indication of scale. The white contours plot the outline of the sunspot as inferred from the average intensity in the continuum between the two $\mathrm{Mg}$ II lines. It should be noted that not all rasters covered the entirety of the FOV plotted in Fig. 1, meaning the FOV plotted in this figure is close to but not perfectly aligned with Fig. 1.

downflow had been detected when the peak intensity of the line was larger than $50 \mathrm{DN}$ in this spectral range and when a Gaussian was returned between $50 \mathrm{~km} \mathrm{~s}^{-1}$ and $150 \mathrm{~km} \mathrm{~s}^{-1}$ with a peak intensity larger than $30 \mathrm{DN}$ and a width (in Doppler units) of larger than $10 \mathrm{~km} \mathrm{~s}^{-1}$. For the O IV $1401 \AA$ line, the required initial thresholding intensity of the line was $30 \mathrm{DN}$, the maximum returned velocity was $100 \mathrm{~km} \mathrm{~s}^{-1}$, and the secondary emission peak was required to have an intensity of over $20 \mathrm{DN}$; we searched for higher peak velocities manually but these values were only detected once and, therefore, this lower peak velocity was implemented to minimise the effects of noise in the fitting. The background transition region intensity of the FOV was estimated through the construction of two-dimensional maps of the SiIV $1394 \AA$ and O IV $1401 \AA$ line intensities at their rest wavelengths.

In Fig. 2, we plot six examples of the SiIV $1394 \AA$ core intensity maps, where each panel corresponds to the respective panel in Fig. 1. An extended bright region is clear in the sunspot stretching above both the umbra and penumbra throughout the time-period analysed; the outline of the umbra as inferred from the $\mathrm{Mg}$ II continuum between the two spectral lines is overlaid in white. The coloured contours outline regions where downflows were detected with aqua indicating a velocity of over $50 \mathrm{~km} \mathrm{~s}^{-1}$ and blue indicating a velocity of over $75 \mathrm{~km} \mathrm{~s}^{-1}$. Applying the typical formation temperatures of the Si IV $1394 \AA$ line $\left(10^{4.8}-10^{5} \mathrm{~K}\right)$ to the formula $C_{\mathrm{s}}=152 \mathrm{~T}^{0.5} \mathrm{~m} \mathrm{~s}^{-1}$ (Priest 1984) allows us to infer that downflow velocities over $50 \mathrm{~km} \mathrm{~s}^{-1}$ are supersonic. It should be noted that the FOV in Fig. 2 and the FOV in Fig. 1 are not perfectly aligned owing to the different rasters sampling slightly different regions around the sunspot with some rasters only partially covering the penumbra. The choice was made to have this slight offset in the FOVs to better emphasise the evolution of the entire spot in Fig. 1.

In Fig. 3, we reproduce Fig. 2 but with the background intensity and velocity contours calculated from the O IV $1401 \AA$ line. It is immediately evident that supersonic downflows in the O IV $1401 \AA$ line covered a larger spatial extent than supersonic downflows in the Si IV $1394 \AA$ line in the top left and top middle panels, and downflows occurred along almost the entirety of the transition region brightening. To quantify this, we calculated the number of pixels within which supersonic downflows over $50 \mathrm{~km} \mathrm{~s}^{-1}$ were detected for both the Si IV $1394 \AA$ and O IV $1401 \AA$ lines (the criteria defined in the second paragraph of this section). In the top left panel, supersonic downflows were detected in 62 and 127 pixels, respectively. Within the top middle panel the corresponding number of pixels for the two lines was 61 and 136. Although such a large disparity in the number of pixels displaying supersonic downflows in the Si IV $1394 \AA$ 


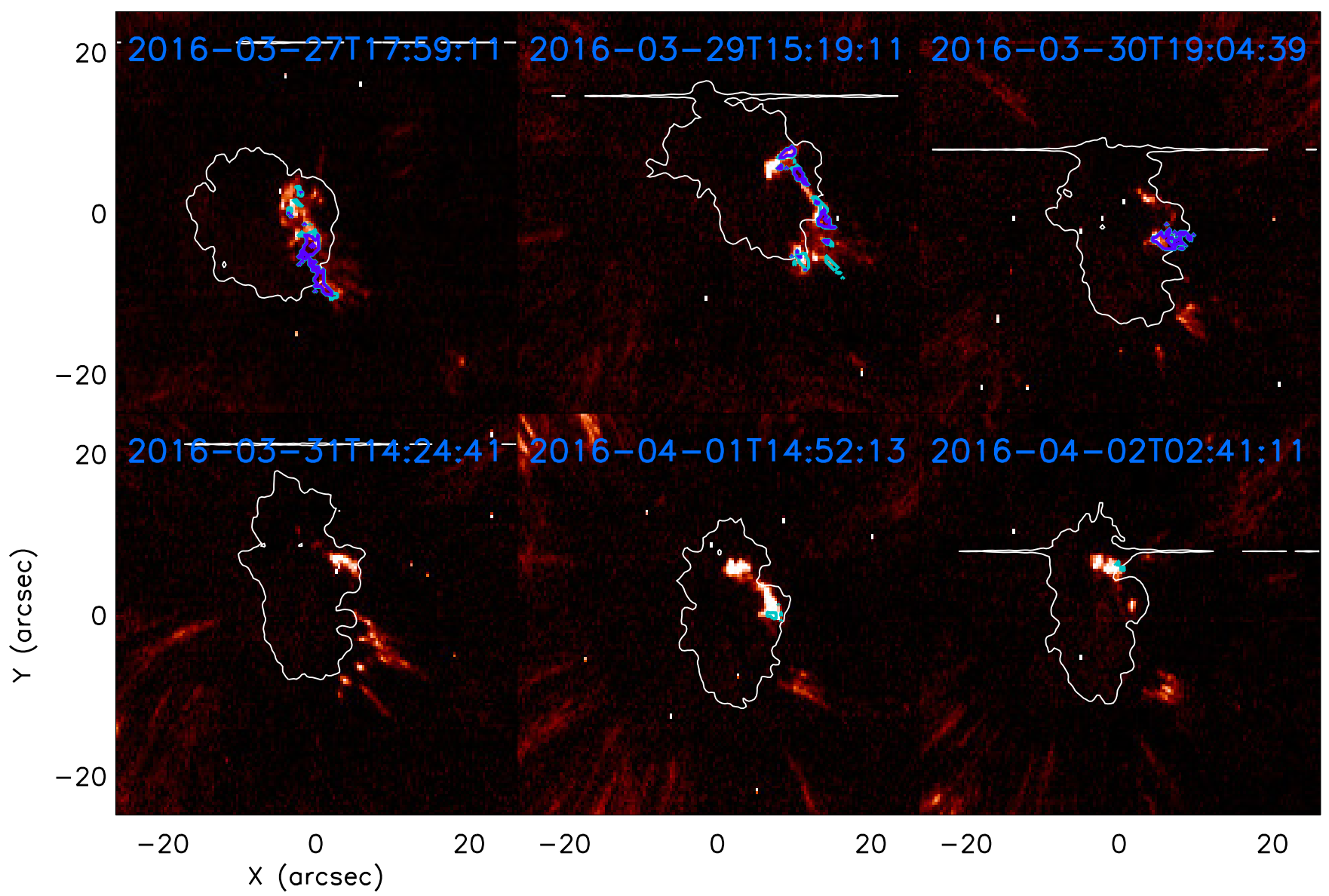

Fig. 3. Same as Fig. 2 but for the O IV $1401 \AA$ line. The spatial coverage of downflows in the top left and top middle panels is larger than for the respective Si IV $1394 \AA$ A plots. Additionally, the bottom right panel displays evidence of a supersonic downflow in the O IV $1401 \AA$ line when no downflow was detected in the Si IV $1394 \AA$ line for this raster.

and O IV $1401 \AA$ lines is not apparent in each raster studied (see final two columns of Table 1), this result confirms that the spatial structuring of these dual flows can be different in different spectral (i.e. thermal) windows. Additionally, the bottom right panel displays evidence of a supersonic downflow in the O IV $1401 \AA$ line, which is not present in the Si IV $1394 \AA$ line, further highlighting the difference in spatial structuring between these wavelengths.

Of the 16 raster scans analysed, 13 (14) displayed evidence of downflows with velocities higher than $50 \mathrm{~km} \mathrm{~s}^{-1}$ in the Si IV $1394 \AA$ (O IV $1401 \AA$ ) line. All detected supersonic downflows formed co-spatial to the intense structure at the centre of the sunspot easily evident in the Si IV $1394 \AA$ line core (see Fig. 2); no dual flows were detected in the sunspot away from this transition region brightening. The evolution of the supersonic downflows through time exhibit no clear pattern; the spatial positioning along the brightening and the sizes of the detected downflows change considerably from raster to raster. To represent this, we plot the peak downflow velocity in the Si IV $1394 \AA$ line and the co-spatial, co-temporal downflow velocities from the O IV $1401 \AA$ line (blue crosses) in Fig. 4. We note that supersonic downflows are not detected in the O IV $1401 \AA$ line at the sites of the peak Si IV $1394 \AA$ downflow velocities in three rasters (see Table 1 for detailed information). The error bars indicate the spectral sampling of the data and the dashed vertical line indicates the approximate formation time of the light bridges.

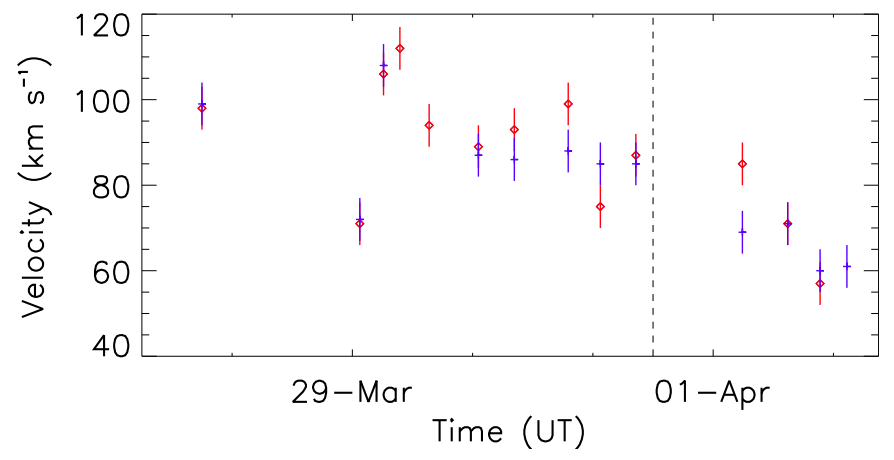

Fig. 4. Peak supersonic downflow velocities inferred from the Si IV $1394 \AA$ (red squares) for each raster. The blue crosses indicate the cospatial O IV $1401 \AA$ downflow velocity. The error bars highlight the spectral sampling and the dashed vertical line indicates the approximate time of the light bridge formation.

Each of the ten datasets sampled prior to the appearance of the light bridges (14:24 on 31 March 2016) displayed some evidence of supersonic downflows in the Si IV $1394 \AA$ line cospatial to the sunspot transition region brightening (see Table 1). Downflow velocities of over $70 \mathrm{~km} \mathrm{~s}^{-1}$ were detected in all of these datasets; velocities of over $100 \mathrm{~km} \mathrm{~s}^{-1}$ were even evident in two rasters (the consecutive scans starting at 06:13 UT and 09:29 UT on 29 March 2016). As the sunspot rotated from $x_{\mathrm{c}}=-595^{\prime \prime}$ to $x_{\mathrm{c}}=171^{\prime \prime}$ during this time we compared the 
projected and deprojected downflow velocities to confirm that the $\mu$-angle of the observation does not affect our results. We found only small change in the estimated downflow velocity of around $5 \mathrm{~km} \mathrm{~s}^{-1}$ (comparable to the spectral sampling). The spatial structuring of the detected dual flows in these rasters was extremely variable; large areas of downflows ( $<65$ pixels) were detected in the Si IV $1394 \AA$ line in five datasets and only small $(<25$ pixels) areas of downflows were evident in the remaining five raster scans.

Following the appearance of the light bridges, however, supersonic downflows became spatially much less prevalent within the sunspot (see the bottom row of Fig. 2). Indeed, all three datasets for which no downflows of over $50 \mathrm{~km} \mathrm{~s}^{-1}$ were detected in the Si IV $1394 \AA$ line occurred following the appearance of the light bridges (the final six rows of Table 1). The supersonic downflows in the further three rasters were extremely localised with spatial extents of only a few pixels $(18,9$, and 7 pixels each in the Si IV $1394 \AA$ line), while only one showed evidence of a supersonic downflow with a velocity of over $75 \mathrm{~km} \mathrm{~s}^{-1}$. Overall, the average peak downflow velocity in the Si IV $1394 \AA$ line prior to the formation of the light bridges is $91.4 \mathrm{~km} \mathrm{~s}^{-1}$ compared to $71 \mathrm{~km} \mathrm{~s}^{-1}$ (calculated from the three rasters which displayed supersonic downflows) after the formation of the light bridges. We cannot confidently comment on whether the change in the supersonic velocities apparent through time is caused by the formation of the light bridges or not because of the small number of scans collected both before and after this time, however, we can conclusively say that the presence of light bridges in a sunspot is not a required condition for the occurrence of supersonic downflows in the transition region (hypothesised by Nicolas et al. 1982).

To further investigate these supersonic downflows, we examined the spectra of the O IV $1401 \AA$, C II $1335 \AA$, and Mg II $2796 \AA$ lines co-spatial to the peak downflow velocity detected in the Si IV $1394 \AA$ A line for each raster (pixel locations included in Table 1). Supersonic downflows were present at these locations in the O IV $1401 \AA$ line for 10 of the 13 datasets, and the velocities (calculated by fitting double Gaussians at these locations) returned are comparable to the velocities inferred from the Si IV $1394 \AA$ line (agreeing with the results of Samanta et al. 2018). The core intensity of the O IV $1401 \AA$ line for two of the datasets in which no supersonic downflows were detected are extremely low, meaning any dual peaks in the line wing may be below the noise level. Additionally, one example of a supersonic downflow, with a peak velocity of $61 \mathrm{~km} \mathrm{~s}^{-1}$, was detected in the O IV $1401 \AA$ line in a raster when no Si IV $1394 \AA$ downflow was apparent (bottom row of Table 1). One example of a downflow was inferred from the C II $1335 \AA$ line; the velocity of $100 \mathrm{~km} \mathrm{~s}^{-1}$ is $15 \mathrm{~km} \mathrm{~s}^{-1}$ faster than the local Si IV $1394 \AA$ downflow (see Table 1). From this one observation we are unable to comment on the generality or significance of this result. No signature of supersonic downflows was detected in the Mg II $2796 \AA$ lines co-spatial to these dual flows.

Finally, we provide a brief overview of the raster sampled prior to the sit-and-stare observations analysed in the following subsection. In the top row of Fig. 5, we plot the intensity of the Si IV $1394 \AA$ line at three different positions within the spectral profile. The sunspot brightening within which all supersonic downflows are detected in this work manifests as an arclike structure in the rest wavelength at this time (top left panel), however, at $31 \mathrm{~km} \mathrm{~s}^{-1}$ (corresponding to approximately $0.15 \AA$ from the line core) only the northern and southern-most regions of the arc-like structure are evident. Further out into the line at approximately $64 \mathrm{~km} \mathrm{~s}^{-1}$ (around $0.3 \AA$ from the line core), only the southern region of the brightening structure has a signature, indicating that no supersonic downflows are present at the northern end of the brightening. In the middle row of Fig. 5 we plot a spectral representation of this from the Si IV $1394 \AA$ line with the intensities taken at the location of the crosses in the top panels. The supersonic downflow is evidenced by the secondary peak in intensity to the right of the location of the second vertical dotdashed line in the right-hand panel. The blue dashed line overlaid plots the Gaussian fit returned for this pixel. The dashed profiles plot the average umbral profile sampled from the blue boxes. The bottom row plots the same as the middle row but for the O IV $1401 \AA$ line. We note that the secondary peak in the O IV $1401 \AA$ line is not large enough to be returned automatically by the algorithm used in this work.

\subsection{Sit-and-stare sequence}

To continue our analysis, we conducted a more detailed investigation of the signatures of supersonic downflows within the sunspot in a sit-and-stare observation sampled on 1 April 2016. In Fig. 6 we plot the FOV at the beginning of the observation, including the SDO/HMI magnetogram and continuum (top left and middle panels), the IRIS chromospheric Mg II $2796 \AA$ and transition region Si IV $1400 \AA$ filters (top right and bottom left panels, respectively), and the SDO/AIA $304 \AA$ and $171 \AA$ channels (bottom middle and right panels). Each panel plots the nearest frame for that channel to $16: 23: 28 \mathrm{UT}$. The blue contours overlaid on Fig. 6 map the brightest regions within the sunspot in the $1400 \AA$ channel, corresponding to the bright arc-like structure plotted in the top left panel of Fig. 5. The blue contours on the SDO/HMI continuum panel confirm that this arc-like brightening stretches across the well-developed northern light bridge. No evidence of this brightening is apparent in the cooler $\mathrm{Mg}$ II $2796 \AA$ filter.

As can be seen in Fig. 6, the IRIS slit passed directly through the northern end of the arc-like brightening during the sit-andstare sequence, away from the location where supersonic downflows were detected in the preceding raster (see Fig. 5). In Fig. 7, we plot spectral-time plots for both the Si IV $1394 \AA$ and the O IV $1401 \AA$ lines for the length of the sit-and-stare observation for a pixel within the brightening. Initially, no supersonic downflow is apparent in either line, agreeing with the spectra sampled for this location during the raster observation (Fig. 5); however, a downflow begins to develop with an initial velocity of $\approx 73 \mathrm{~km} \mathrm{~s}^{-1}$ in both the Si IV $1394 \AA$ and O IV $1401 \AA$ lines after around $60 \mathrm{~min}$ (corresponding to approximately 17:25 UT). The downflow then accelerates at approximately $5 \mathrm{~m} \mathrm{~s}^{-2}$ to a velocity of $\approx 80 \mathrm{~km} \mathrm{~s}^{-1}$ over the course of the remaining $\sim 20 \mathrm{~min}$, indicated by the dashed blue line.

In the top left and top middle panels of Fig. 8, we plot the sunspot as sampled by the IRIS SJI $1400 \AA$ filter in both the first and last frames. The arc-like brightening is relatively stable during this time, and only a small increase in brightness and size is detected at the north end over the course of the $80 \mathrm{~min}$. Plotting light curves of these increases in size and brightness (not shown here) demonstrates that they occur relatively consistently throughout the observations and are continuously modulated by the typical three-minute sunspot oscillations. Additionally, the ratio of the Si IV $1394 \AA$ and $1403 \AA$ lines within the arc-like brightening throughout the time-series 

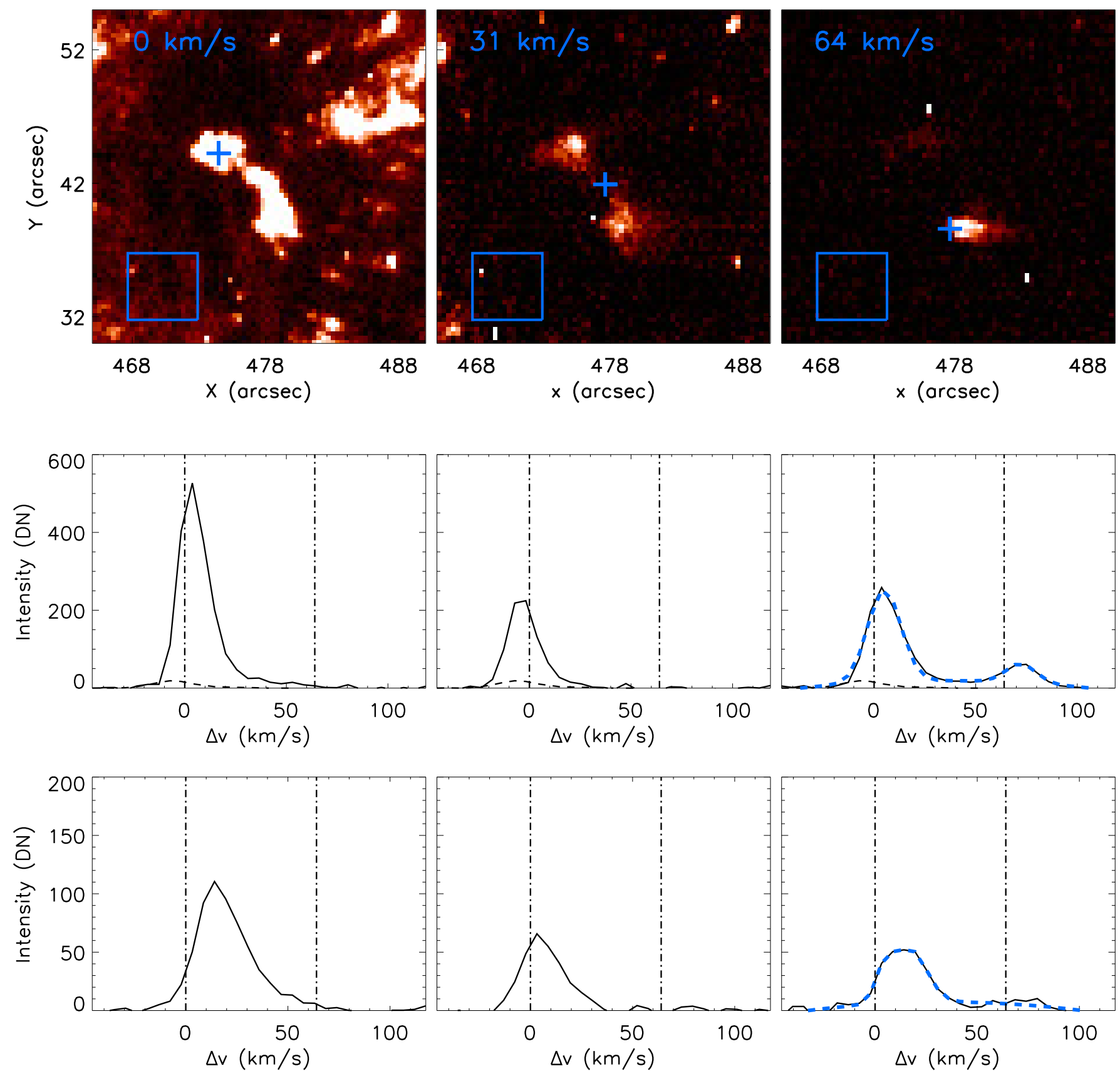

Fig. 5. Top row: Si IV $1394 \AA$ Antensity maps at three different positions within the line profile for the raster beginning at 14:52 on 1 April 2016 . The LOS velocities corresponding to the specific line positions are indicated in the top left of each panel. Middle row: Si IV $1394 \AA$ spectra for the spatial positions indicated by the crosses in the top row. Bottom row: same as the middle row but for the O IV $1401 \AA$ line. Average spectra from the sunspot umbra, calculated from the boxes in the bottom left of the images in the top row, are overplotted as dashed lines. The vertical lines indicate the rest velocity of the lines and a LOS velocity of $64 \mathrm{~km} \mathrm{~s}^{-1}$ (matching the line position plotted in the top right panel). The dashed blue lines on the right hand panels of the bottom two rows plot the double Gaussian fits for this pixel. As previously reported in Table 1, no clear evidence of a secondary emission peak is found in the O IV $1401 \AA$ line at this location.

remains close to 2, implying no changes in the opacity of the lines occurs through time (Mathioudakis et al. 1999). Overall, no large-scale morphological changes are evident in the arc-like brightening during this time despite the onset of the supersonic downflow.

In the other four panels of Fig. 8, the spectra from the second and second-to-last slit exposures (black and blue lines, respectively) are plotted. For the $\mathrm{C}$ II and $\mathrm{Mg}$ II lines (top right and bottom left panels), increases in intensity of around 10-30\% are detected between the blue and black profiles, however, no secondary peak associated with supersonic downflows is evident. This is in agreement with the lack of footpoint signature in the chromospheric Mg II $2796 \AA$ SJI data (see top right panel of Fig. 6). For the Si IV and O IV lines (bottom middle and bottom left panels), no peaks associated with supersonic downflows are present in the black spectra, however, such peaks are present in the blue spectra with approximate velocities of $80 \mathrm{~km} \mathrm{~s}^{-1}$. No signature of this downflow was evident in the raster scan starting around $100 \mathrm{~min}$ following the end of the sit-and-stare observation. 


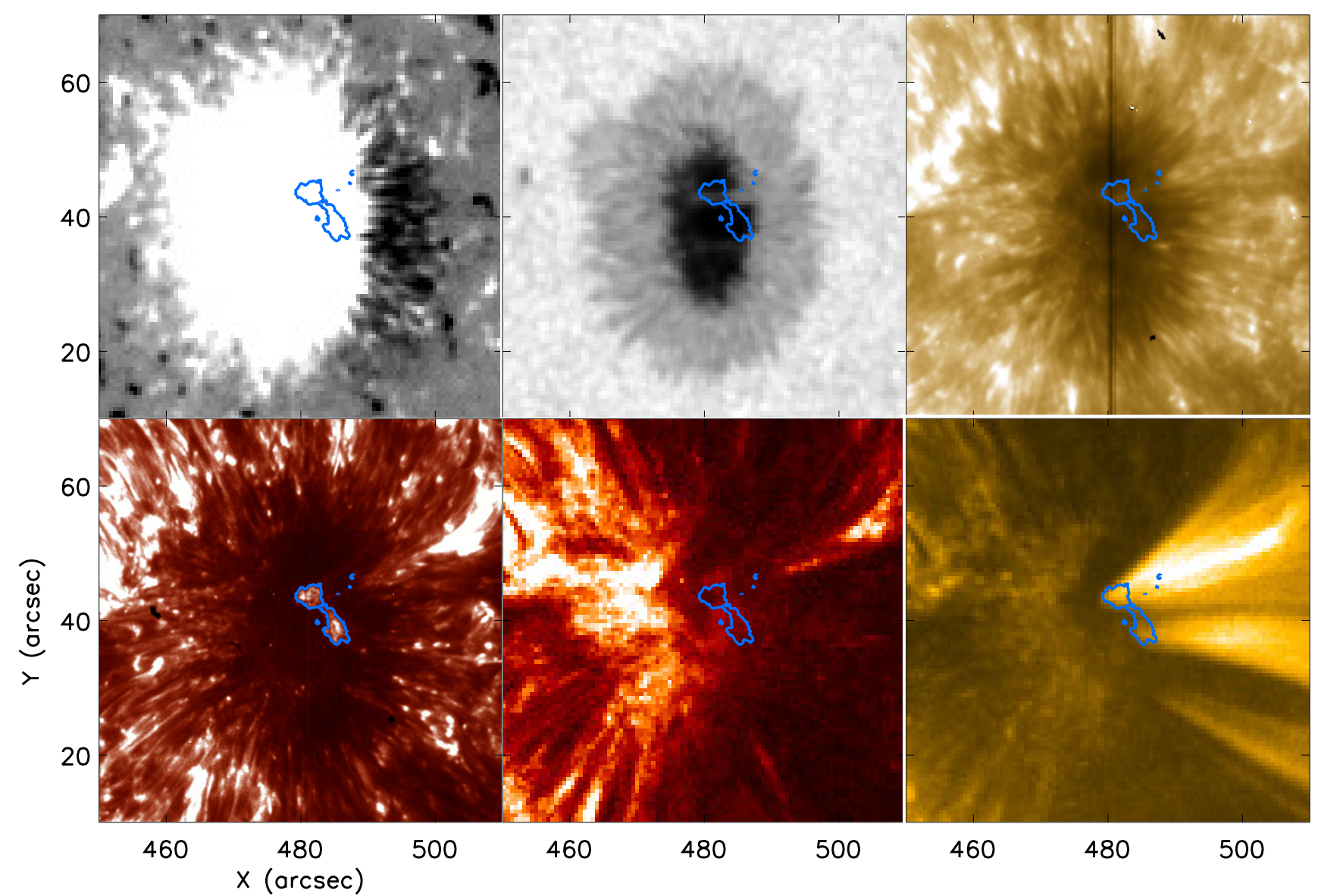

Fig. 6. Sunspot within AR 12526 at approximately 16:23:28 UT on 1 April 2016. The photospheric LOS magnetic field and continuum intensity (top left and middle panels) sampled by the SDO/HMI instrument display a large positive polarity sunspot. The solar atmosphere above the sunspot was sampled using the IRIS Mg II $2796 \AA$ (top right) and Si IV $1400 \AA$ (bottom left) SJI filters, as well as the SDO/AIA $304 \AA$ (bottom middle) and $171 \AA$ (bottom right) channels. The IRIS slit position during the sit-and-stare sequence passes directly through the sunspot and is most easily evident in the $2796 \AA$ image. The blue contours outline the bright feature in the IRIS $1400 \AA$ channel within which all supersonic downflows are detected in this sunspot.

Interestingly, the arc-like brightening in the transition region coincides spatially with the footpoints of two apparently distinct coronal fan loops in the $171 \AA$ A filter (see Fig. 6). A clear separation appears to be present between the two distinct coronal fan loops, such that one is anchored in the northern section of the umbra and one is anchored in the southern section of the umbra. A clear gap between these two structures extends to the edge of the FOV (the darker horizontal line at $y \approx 42^{\prime \prime}$ ) and beyond. Although it is difficult to track them along their entire lengths, it is possible that the other ends of these two loop systems are rooted in completely separate locations within the active region with the northern fan loops appearing to be rooted in the trailing plage region and the southern fan loops potentially rooted in the opposite polarity structures leading the sunspot. Future analysis using non-linear force free magnetic field extrapolations could better map the connectivity of the active region and could, therefore, provide more evidence about where these loops are anchored. Such research could allow us to better examine the potential causes of these downflows.

\section{Discussion and conclusions}

In Table 1 , the basic properties of the 16 datasets analysed in this work are presented. Overall, 13 of the 16 datasets include evidence of supersonic downflows in the Si IV $1394 \AA$ A line (a similar ratio to that found by Samanta et al. 2018); a peak velocity of $112 \mathrm{~km} \mathrm{~s}^{-1}$ was found on 29 March 2016. In Fig. 2, the transition region intensity estimated by the rest wavelength of the Si IV $1394 \AA$ A line is plotted and contours of downflow velocities with magnitudes of $50 \mathrm{~km} \mathrm{~s}^{-1}$ (aqua) and $75 \mathrm{~km} \mathrm{~s}^{-1}$ (blue) are overlaid. Fig. 3 plots the equivalent as calculated from the O IV 1401 Å line. All supersonic downflows detected within the sunspot occur co-spatial to a sustained transition region brightening spanning across the umbra and penumbra. This transition region brightening appears to map the locations of coronal loop foot-points, as observed in the SDO/AIA $171 \AA$ A channel. The spatial positioning of supersonic downflows through time is highly variable; some rasters reveal relatively large-scale downflows across the entire transition region brightening and some rasters reveal only point-like downflows. Additionally, the spatial coverage of dual flows in the O IV $1401 \AA$ line is often different to the spatial coverage in the Si IV $1394 \AA$ line, with the spatial coverage in the O IV $1401 \AA$ line sometimes being double that of the Si IV $1394 \AA$ line.

From both Table 1 and Fig. 4, it is immediately evident that the peak velocity of supersonic downflows within the umbra is variable through time. This result is in agreement with previous temporal analyses of supersonic downflows within sunspots 


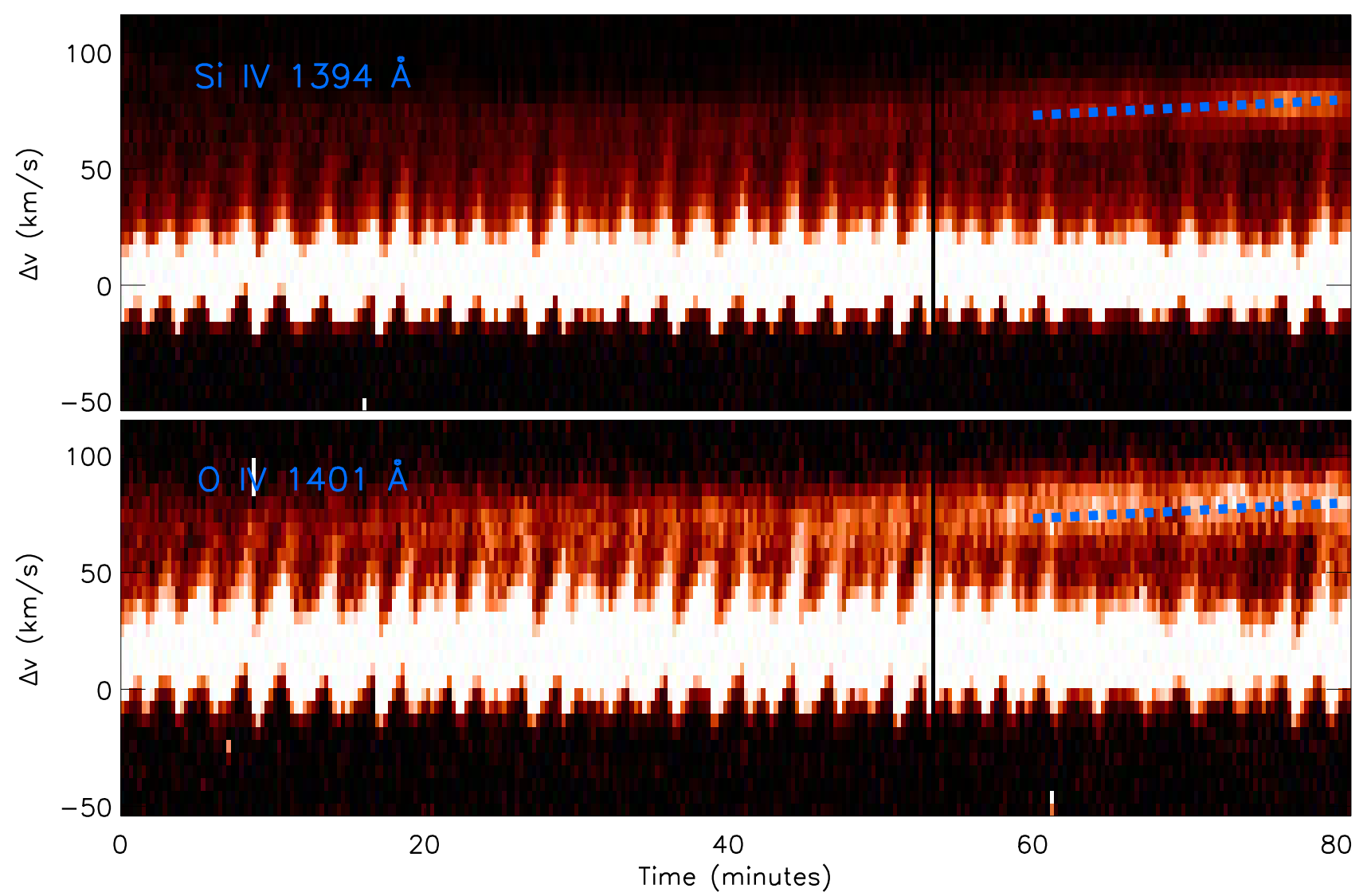

Fig. 7. Top row: spectral-time plot for the Si IV $1394 \AA$ Aine for a pixel within the transition region brightening. The typical saw-tooth shock pattern is clearly evident close to the line core. The supersonic downflow can be detected from around 60 min until the end of the time series. During this time, the flow accelerates at approximately $5 \mathrm{~m} \mathrm{~s}^{-2}$ from around $73 \mathrm{~km} \mathrm{~s}^{-1}$ to close to $80 \mathrm{~km} \mathrm{~s}^{-1}$, as indicated by the dashed blue line. Bottom row: same as the top row except for the O IV $1401 \AA$ line.

(Kjeldseth-Moe et al. 1988) and suggests that single measurements of an AR are unable to provide general information about the properties of supersonic downflows. The average supersonic downflow velocity in the Si IV $1394 \AA$ line, in rasters where such downflows are observed, is calculated to be $86.7 \mathrm{~km} \mathrm{~s}^{-1}$ (consistent with previous measurements using IRIS data by e.g. Straus et al. 2015; Chitta et al. 2016; Samanta et al. 2018). Interestingly, supersonic downflows have been detected both before and after the formation of light bridges in the sunspot, indicating that light bridges in the umbra are not an essential requirement for the formation of supersonic downflows in transition region spectra (as speculated by Nicolas et al. 1982).

Supersonic downflows were also detected in the O IV $1401 \AA$ line at the same spatial locations as the peak Si IV $1394 \AA$ downflow velocity in 10 of the 13 rasters (pixel co-ordinates in Table 1). For the other three rasters, downflows were apparent in the O IV $1401 \AA$ in the surrounding pixels again highlighting the differences in spatial structuring between these two lines. Additionally, one raster displayed evidence of a supersonic downflow in the O IV $1401 \AA$ when no downflow was apparent in the Si IV $1394 \AA$ line. As has been found in previous analyses (see e.g. Samanta et al. 2018), the downflow velocities in the O IV $1401 \AA$ line were similar to those inferred from the Si IV $1394 \AA$ line. One example of a supersonic downflow in the C II $1334 \AA$ line was detected at these locations, with the estimated downflow velocity of this line being $15 \mathrm{~km} \mathrm{~s}^{-1}$ higher than the downflow inferred from the Si IV $1394 \AA$ Aine at the same position. No signature was detected in the Mg II $2796 \AA$ lines at any of these locations. Overall, our results could be interpreted as evidence for the presence of multi-thermal, multi-threaded loops (see, for example, Dere 1982; Nicolas et al. 1982), however, they do not rule out other possibilities. If these spectral lines are forming at different heights in the solar atmosphere, one possible interpretation could be that we are observing the acceleration of material down from the hotter regions (Si IV and O IV lines) to the cooler chromospheric regions (C II lines). This would have to be examined further using a range of techniques including $\mathrm{RH}$ modelling (Uitenbroek 2001).

Using an $82 \mathrm{~min}$ sit-and-stare dataset, we were able to analyse the shorter-scale evolution of supersonic downflows within the bright sunspot structure. The IRIS slit passed directly through the northern end of the Si IV transition region brightening that hosted the supersonic downflows analysed in this work, which had no signature in the $\mathrm{Mg}$ II filter, at a position where no supersonic downflows were detected in the previous raster (as shown in Fig. 5). Initially, no downflow was apparent in the Si IV $1394 \AA$ A spectra, however, after approximately 60 min a dual flow developed with an initial velocity of $73 \mathrm{~km} \mathrm{~s}^{-1}$. This downflow then accelerated at a rate of $5 \mathrm{~km} \mathrm{~s}^{-2}$ over the course of the next $20 \mathrm{~min}$ to a velocity of around $80 \mathrm{~km} \mathrm{~s}^{-1}$, similar to the acceleration rate measured at the footpoint of a coronal loop (Chitta et al. 2016). In the O IV $1401 \AA$ spectra, the downflow displayed similar behaviour during the same time period. It is unclear whether this downflow becomes stable at some point (analogous to the downflow studied by Straus et al. 2015) due to the end of the 

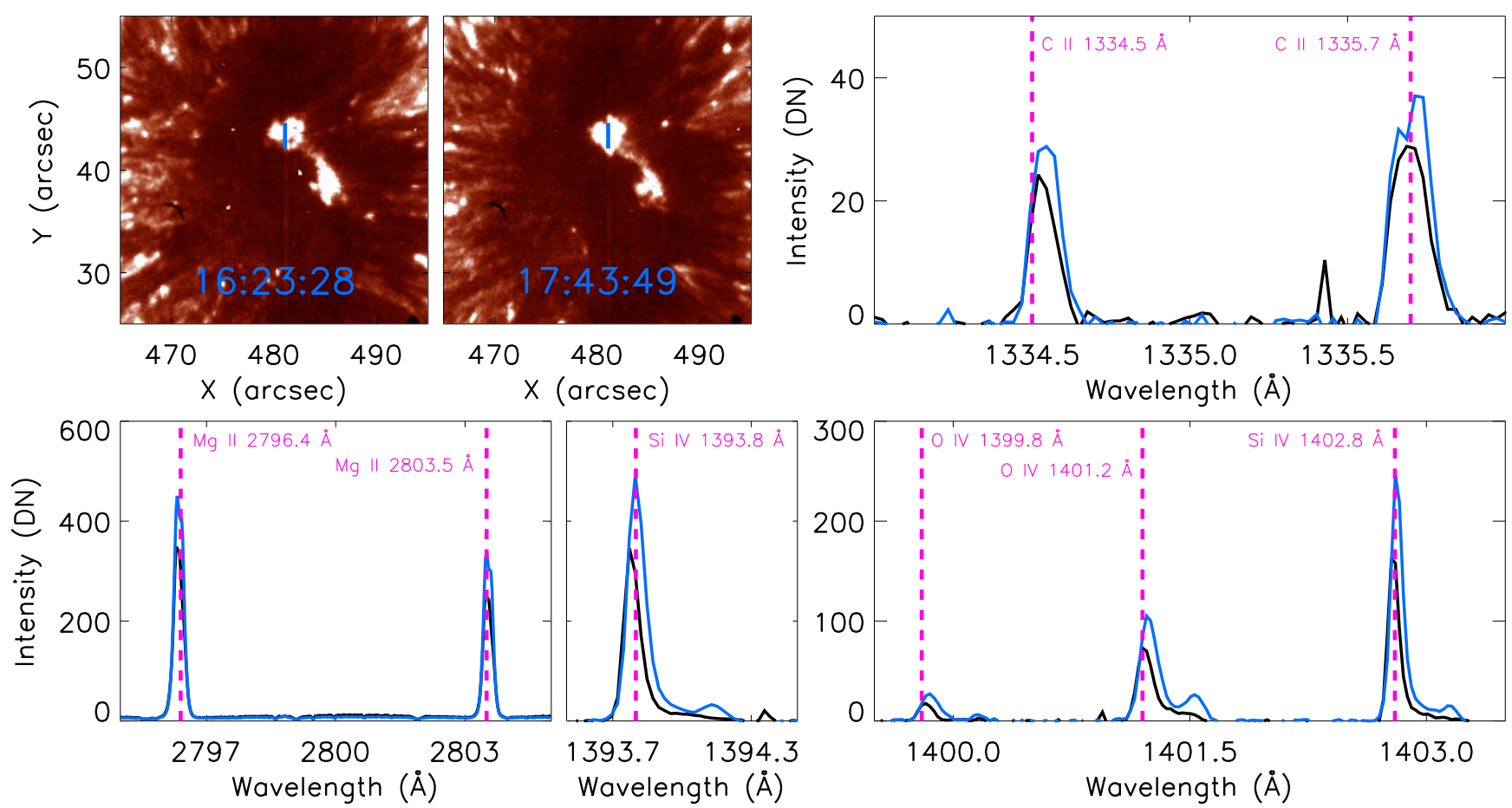

Fig. 8. Evolution of the SJI and spectral data through time. The first and final SJI frames (top left and top middle panels) show that the transition region brightening remained relatively stable with only a small increase in the size of the northern brightening. The blue vertical lines indicate the slits over which the spectra were averaged in the remaining panels. Spectra from the second and second-to-last raster steps (black and blue lines, respectively) display an increase in intensity in all pass-bands during the supersonic downflow, however, only the O IV and Si IV lines possess the second peak in intensity in the red wing indicative of supersonic downflows. The vertical dashed pink lines indicate the rest wavelengths of the lines of interest.

sit-and-stare observation. It should be noted that no evidence of this downflow is present in the raster following the sit-and-stare, indicating that it has a lifetime of only a few hours at most.

Now, we try to place our results in the context of current understanding of the transition region dynamics of sunspots. Although the intensity of the line core is often larger than the background intensity during these downflows, the specific cases where the line core intensity is reduced (see Fig. 5), the prevalence of these signatures in the umbra, and the presence of the secondary emission peak in the red wing appears to differentiate the downflows studied in this work from bright dots (Tian et al. 2014b). The sustained nature of the supersonic downflow in the sit-and-stare observation, the relatively low downflow velocity, and the lack of signature in the C II $1335 \AA$ and Mg II $2796 \AA$ lines for all but one of the detected dual flows, also makes it unlikely that these downflows are a result of bursty events such as coronal rain (Kleint et al. 2014). The supersonic downflows reported in this paper appear to be more similar in velocity and spectral signature to the lower velocity downflows reported in the IRIS literature by several authors (e.g. Tian et al. 2014a; Straus et al. 2015; Chitta et al. 2016; Samanta et al. 2018). It has been suggested that such dual flow signatures in the transition region spectra could be caused by shocks from downflowing material (Brynildsen et al. 2001), potentially, due to siphon flows (Cargill \& Priest 1980). If this is the case then our results imply that the conditions required for siphon flows must be common within ARs throughout their lifetimes.

\section{Summary}

Downflows of over $50 \mathrm{~km} \mathrm{~s}^{-1}$ were present within the lead sunspot of AR 12526 in the Si IV $1394 \AA$ line for 13 of the 16 raster datasets studied in this work (see Table 1). Supersonic downflows were present in the O IV $1401 \AA$ line for 14 of these datasets. All such downflows occurred co-spatial to a sustained transition region brightening, mapping the foot-points of coronal loops detectable in the SDO/AIA $171 \AA$ channel, stretching over the umbra and penumbra. The spatial extents of the downflows in the Si IV $1394 \AA$ and O IV $1401 \AA$ lines was often very different (Figs. 2 and 3). The three rasters which contained no evidence of supersonic downflows within the Si IV $1394 \AA$ line occurred following the formation of two lightbridges within the umbra. Only one example of supersonic downflows was detected in the C II $1335 \AA$ line with a velocity $15 \mathrm{~km} \mathrm{~s}^{-1}$ larger than the corresponding Si IV $1394 \AA$ velocity. No downflows were detected in the Mg II $2796 \AA$ line.

During the sit-and-stare sequence sampled on 1 April 2016, no supersonic downflow was initially present (Figs. 5 and 7). However, a dual flow did form around $60 \mathrm{~min}$ after the start of the observation with an initial velocity of $73 \mathrm{~km} \mathrm{~s}^{-1}$ in the Si IV $1394 \AA$ line. The velocity of this downflow then increased at around $5 \mathrm{~m} \mathrm{~s}^{-2}$ over the course of the next $20 \mathrm{~min}$ to give a final velocity of close to $80 \mathrm{~km} \mathrm{~s}^{-1}$ (top panel of Fig. 7). In the O IV $1401 \AA$ line, the velocity accelerated at a similar rate during the same time. No signature of this downflow was present in either the C II $1335 \AA$ or Mg II $2796 \AA$ lines (Fig. 8). Aligning the SJI images to SDO/AIA and SDO/HMI data allowed us to infer that the brightening over which all supersonic downflows were detected in the rasters appeared to stretch over the northern light bridge at this time. Additionally, two distinct sets of coronal fan loops were evident with one set appearing to be rooted to the north of this light bridge and one set appearing to be rooted south of this light bridge (Fig. 6). 


\section{J. Nelson et al.: Onset of supersonic downflows in a coronal fan loop}

Overall, our results indicate that supersonic downflows are highly irregular and intermittent over the course of both minutes and hours (agreeing with previous research by KjeldsethMoe et al. 1988). The spatial locations and velocities of these downflows, which are most easily detected in the red wing of the SiIV $1394 \AA$ and OIV $1401 \AA$ lines, are often completely different from one raster to the next, and from one spectral window to another. Our analysis also suggests that supersonic downflows occur preferentially around bright regions at the Si IV $1394 \AA$ rest wavelength corresponding to the locations of footpoints of coronal loops. Finally, the intermittency of supersonic downflows implies that more sunspots than previously thought could host such events (i.e. more than the $\sim 80 \%$ reported by Samanta et al. 2018).

Acknowledgements. We thank the Science and Technology Facilities Council (STFC) for the support received to conduct this research through grant number: ST/P000304/1. SKP is grateful to the FWO Vlaanderen for a senior postdoctoral fellowship. IRIS is a NASA small explorer mission developed and operated by LMSAL with mission operations executed at NASA Ames Research Center and major contributions to downlink communications funded by ESA and the Norwegian Space Centre. SDO/HMI and SDO/AIA data are courtesy of NASA/SDO and the HMI and AIA science teams.

\section{References}

Beckers, J. M., \& Tallant, P. E. 1969, Sol. Phys., 7, 351

Bose, S., Henriques, V. M. J., Rouppe van der Voort, L., \& Pereira, T. M. D. 2019, A\&A, 627, A46

Brynildsen, N., Maltby, P., Kjeldseth-Moe, O., \& Wilhelm, K. 2001, ApJ, 552, L77

Brynildsen, N., Maltby, P., Kjeldseth-Moe, O., \& Wilhelm, K. 2004, ApJ, 612, 1193
Cargill, P. J., \& Priest, E. R. 1980, Sol. Phys., 65, 251

Chitta, L. P., Peter, H., \& Young, P. R. 2016, A\&A, 587, A20

De Pontieu, B., Title, A. M., Lemen, J. R., et al. 2014, Sol. Phys., 289, 2733

Dere, K. P. 1982, Sol. Phys., 77, 77

Fludra, A. 2001, A\&A, 368, 639

Hale, G. E. 1908, ApJ, 28, 315

Henriques, V. M. J., Mathioudakis, M., Socas-Navarro, H., \& de la Cruz Rodríguez, J. 2017, ApJ, 845, 102

Jess, D. B., De Moortel, I., Mathioudakis, M., et al. 2012, ApJ, 757, 160

Khomenko, E., \& Collados, M. 2015, Liv. Rev. Sol. Phys., 12, 6

Kjeldseth-Moe, O., Brynildsen, N., Brekke, P., et al. 1988, ApJ, 334, 1066

Kleint, L., Antolin, P., Tian, H., et al. 2014, ApJ, 789, L42

Lagg, A., Woch, J., Solanki, S. K., \& Krupp, N. 2007, A\&A, 462, 1147

Lemen, J. R., Title, A. M., Akin, D. J., et al. 2012, Sol. Phys., 275, 17

Mathioudakis, M., McKenny, J., Keenan, F. P., Williams, D. R., \& Phillips, K. J. H. 1999, A\&A, 351, L23

Nelson, C. J., Henriques, V. M. J., Mathioudakis, M., \& Keenan, F. P. 2017, A\&A, 605, A14

Nicolas, K. R., Bartoe, J. D. F., Brueckner, G. E., \& Kjeldseth-Moe, O. 1982, Sol. Phys., 81, 253

Priest, E. R. 1984, Solar Magneto-hydrodynamics (Dordrecht: Reidel)

Rouppe van der Voort, L., \& de la Cruz Rodríguez, J. 2013, ApJ, 776, 56

Rouppe van der Voort, L. H. M., Rutten, R. J., Sütterlin, P., Sloover, P. J., \& Krijger, J. M. 2003, A\&A, 403, 277

Samanta, T., Tian, H., \& Prasad Choudhary, D. 2018, ApJ, 859, 158

Scherrer, P. H., Schou, J., Bush, R. I., et al. 2012, Sol. Phys., 275, 207

Socas-Navarro, H., de la Cruz Rodríguez, J., Asensio Ramos, A., Trujillo Bueno, J., \& Ruiz Cobo, B. 2015, A\&A, 577, A7

Solanki, S. K. 2003, A\&ARv, 11, 153

Straus, T., Fleck, B., \& Andretta, V. 2015, A\&A, 582, A116

Sych, R., \& Nakariakov, V. M. 2014, A\&A, 569, A72

Tian, H., DeLuca, E., Reeves, K. K., et al. 2014a, ApJ, 786, 137

Tian, H., Kleint, L., Peter, H., et al. 2014b, ApJ, 790, L29

Tian, H., Samanta, T., \& Zhang, J. 2018, Geosci. Lett., 5, 4

Uitenbroek, H. 2001, ApJ, 557, 389

Wittmann, A. 1969, Sol. Phys., 7, 366 